\section{IMPACTO Y ADHERENCIA DE LA SUPLEMENTACIÓN CON MULTIMICRONUTRIENTES EN NIÑOS DE PERÚ}

\author{
IMPACT AND ADHERENCE OF MULTI- \\ MICRONUTRIENT SUPPLEMENTATION IN \\ PERUVIAN CHILDREN
}

\author{
Sebastián Trelles ${ }^{1, a}$, Cesar V. Munayco ${ }^{1,2, b}$
}

Sr. Editor. La suplementación con multimicronutrientes ha demostrado ser una forma efectiva de disminuir la prevalencia de anemia en niños, siempre y cuando se tenga una buena adherencia a la misma ${ }^{(1,2)}$. Según el reporte del Instituto de Nacional de Estadística e Informática del Perú, la adherencia promedio a la suplementación con hierro en los últimos cinco años ha sido de $23,3 \%{ }^{\left({ }^{3}\right)}$, lo que explicaría el poco cambio que se ha visto en la tendencia de la prevalencia de anemia en los últimos años, ya que ha permanecido constante alrededor de $43 \%{ }^{(3)}$.

Cuando se introdujeron los micronutrientes en el Perú, como una nueva estrategia para derrotar la anemia en niños de 6 a 35 meses de edad, el esquema de suplementación era dar los micronutrientes de manera interdiaria por seis meses para después descansar seis meses, y luego continuar seis meses más de forma interdiaria. Este esquema resultó ser efectivo, como lo demostraron los resultados de la vigilancia centinela del Centro Nacional de Epidemiología, Prevención y Control de Enfermedades del Perú (1).

El objetivo de esta comunicación es mostrar el impacto de la suplementación de hierrocon micronutrientes sobrelos valores de hemoglobina durante todo el periodo de suplementación, además de determinar los cambios en la adherencia a la suplementación con multimicronutrientes teniendo en cuenta la variabilidad intra sujetos de estudio (medidas repetidas y correlación) y los valores perdidos a través de un modelo de ecuaciones de estimación generalizadas (GEE por sus siglas en inglés) ${ }^{(4)}$.

Los datos son parte del análisis de datos de la vigilancia centinela realizada por CDC Perú en 29 centros de salud de Ayacucho, Huancavelica y Apurímac. Esta vigilancia se realizó en niños y niñas de 6 a 35 meses de edad durante 2009 al 2011. Esta vigilancia centinela tuvo un diseño de cohortes prospectiva, donde se siguieron a los niños

\footnotetext{
1 Escuela de Medicina, Universidad Peruana de Ciencias Aplicadas. Lima, Perú.

2 Centro Nacional de Epidemiología Prevención y Control de Enfermedades, Ministerio de Salud. Lima, Perú.

a Estudiante de Medicina; ${ }^{b}$ Médico, magíster en Epidemiología y salud pública Recibido: 14/11/2018 Aprobado:30/01/2019 En línea: 11/03/2019
}

Citar como: Trelles S, Munayco CV. Impacto y adherencia de la suplementación con multimicronutrientes en niños de Perú. Rev Peru Med Exp Salud Publica. 2019;36(1):147-8. doi:10.17843/rpmesp.2019.361.4051. durante tres periodos: dos periodos de suplementación de seis meses con un periodo de descanso de seis meses entre el primer y segundo periodo de suplementación. Además, se midieron los valores de hemoglobina al inicio y final de cada periodo de suplementación y la adherencia mensual por conteo de sobres consumidos en el mes ${ }^{(1)}$.

Los dos modelos GEE para la hemoglobina y adherencia a la suplementación se ajustaron por las áreas de estudio. En la Tabla 1 se observa que el coeficiente beta de la regresión GEE se incrementó 0,38 con respecto a la línea basal al final de los seis primeros meses de suplementación, pero desciende como es de esperar después del periodo de descanso. Además, el coeficiente beta, al final del segundo periodo de suplementación, se duplicó comparado con el coeficiente beta al final del primer periodo de suplementación. Todos estos coeficientes fueron estadísticamente significativos.

El coeficiente beta de la adherencia a la suplementación con multimicronutrientes se incrementó mes a mes con respecto a la línea basal. Este incremento fue significativo a partir del cuarto mes de suplementación, alcanzando un valor de 3,69 al final de la suplementación. Se aprecia un incremento considerable después del periodo de descanso (Tabla 1).

Los resultados de este estudio muestran que hubo una mejora importante en los niveles de hemoglobina con respecto a la línea basal, lo cual probablemente se deba a la alta adherencia. Todo lo contrario, se viene observando en las Encuestas Demográficas y de Salud Familiar, donde la adherencia sólo llega al $23 \%{ }^{(3)}$. Además, se muestra que la adherencia a la suplementación con multimicronutrientes se incrementó significativamente mes a mes durante todo el periodo de suplementación.

Esta alta adherencia a la suplementación con multimicronutrientes podría haberse explicado por el hecho de que las madres debían acudir mensualmente con sus niños a los establecimientos centinelas para entregar los sobres vacíos de los multimicronutrientes al personal de salud. De acuerdo con la norma técnica de crecimiento y desarrollo (CRED), sólo los niños menores de un año acuden mensualmente al control CRED, y los mayores de un año lo hacen cada tres meses ${ }^{(5)}$.

Probablemente el seguimiento cercano, como el que se tuvo en este estudio, sea un factor importante para mantener valores de adherencia altas, inclusive después de un periodo de descanso. Finalmente, este estudio da más evidencias de que los multimicronutrientes son efectivos si se garantiza una buena adherencia, y que el seguimiento cercano podría ser un factor clave para mantener adherencias altas.

Contribuciones de autoría: ST y CVM han participado en la concepción y diseño del artículo, su redacción y aprobación de la versión final.

Financiamiento: Esta carta fue autofinanciada.

Conflictos de interés: No hay conflictos de interés. 
Tabla 1. Suplementación con micronutrientes sobre los valores de hemoglobina según impacto y variación de la adherencia

\begin{tabular}{|c|c|c|c|c|}
\hline & Coeficiente beta & Error estándar & Valor de $p$ & IC $95 \%$ \\
\hline \multicolumn{5}{|l|}{ Impacto } \\
\hline $\mathrm{Hb}$ inicio de primera entrega (t0) & referencia & & & \\
\hline $\mathrm{Hb}$ final primera entrega (t1) & 0,38 & 0,42 & $<0,001$ & 0,30 a 0,46 \\
\hline $\mathrm{Hb}$ inicio segunda entrega (t2) & 0,28 & 0,45 & $<0,001$ & 0,19 a 0,37 \\
\hline $\mathrm{Hb}$ final segunda entrega ( $\mathrm{t} 3$ ) & 0,74 & 0,48 & $<0,001$ & 0,65 a 0,84 \\
\hline Constante & 10,17 & 0,06 & $<0,001$ & 10,05 a 10,29 \\
\hline \multicolumn{5}{|l|}{ Variación de la adherencia } \\
\hline Adherencia en el mes 1 (T0) & referencia & & & \\
\hline Adherencia en el mes 2 (T1) & 0,33 & 0,31 & 0,28 & $-0,27$ a 0,93 \\
\hline Adherencia en el mes 3 (T2) & 0,56 & 0,31 & 0,07 & $-0,04$ a 1,17 \\
\hline Adherencia en el mes 4 (T3) & 0,87 & 0,31 & 0,005 & 0,26 a 1,48 \\
\hline Adherencia en el mes 5 (T4) & 1,28 & 0,31 & $<0,001$ & 0,67 a 1,90 \\
\hline Adherencia en el mes 6 (T5) & 1,72 & 0,32 & $<0,001$ & 1,10 a 2,35 \\
\hline Adherencia en el mes 13 (T6) & 2,66 & 0,35 & $<0,001$ & 1,98 a 3,34 \\
\hline Adherencia en el mes 14 (T7) & 3,16 & 0,35 & $<0,001$ & 2,47 a 3,85 \\
\hline Adherencia en el mes 15 (T8) & 3,27 & 0,36 & $<0,001$ & 2,57 a 3,97 \\
\hline Adherencia en el mes 16 (T9) & 3,50 & 0,36 & $<0,001$ & 2,80 a 4,21 \\
\hline Adherencia en el mes 17 (T10) & 3,56 & 0,36 & $<0,001$ & 2,84 a 4,27 \\
\hline Adherencia en el mes 18 (T11) & 3,69 & 0,37 & $<0,001$ & 2,97 a 4,41 \\
\hline Constante & 93,10 & 0,44 & $<0,001$ & 92,23 а 93,96 \\
\hline
\end{tabular}

\section{REFERENCIAS BIBLIOGRÁFICAS}

1. Munayco CV, Ulloa-Rea ME, Medina-Osis J, LozanoRevollar CR, Tejada V, Castro-Salazar C, et al. Evaluación del impacto de los multimicronutrientes en polvo sobre la anemia infantil en tres regiones andinas del Perú. Rev Peru Med Exp Salud Publica. 2013;30(2):229-34.

2. Huamán-Espino L, Aparco JP, Nuñez-Robles E, Gonzáles E, Pillaca J, Mayta-Tristán P. Consumo de suplementos con multimicronutrientes Chispitas y anemia en niños de 6 a 35 meses: estudio transversal en el contexto de una intervención poblacional en Apurímac, Perú. Rev Peru Med Exp Salud Publica. 2012;29(3): 314-323.

3. Instituto Nacional de Estadistica e Informatica. Indicadores de Resultados de los Programas Presupuestales, Primer Semestre 2017 Encuesta Demográfica y de Salud Familiar (Resultados Preliminares) [Internet]. Lima; 2017. [citado el 16 de agosto de 2018. Disponible en: https://proyectos.inei.gob. pe/endes/images/Indicadores_Resultados_PPR_Primer_ Semestre_2017.pdf.

4. Arnau J. Estudios longitudinales de medidas repetidas. Modelos de diseño y de análisis. Av en Medicin. 2007;5(1):9-26.

5. Ministerio de Salud. Norma Técnica de Salud para el Control del Crecimiento y Desarrollo de la Niña y el Niño menor de cinco años. Resolución Ministerial Nº 990 - 2010/MINSA. 2011.

\section{Correspondencia: Sebastián Trelles}

Dirección: Teodosio Parreño 341 dpto 203

Correo:setzal@yahoo.com.pe

\section{LEPTOSPIROSIS Y COINFECCIONES DURANTE EL NIÑO COSTERO EN UN HOSPITAL DEL NORTE PERUANO}

\section{LEPTOSPIROSIS AND CO-INFECTIONS DURING THE COASTAL "EL NIÑO" IN A HOSPITAL IN NORTHERN PERU}

\section{Dennis Herrera-Pérez ${ }^{1, a}$, Alexandra Saavedra-Barón ${ }^{1, a}$, Jorge Fernández-Mogollón 1,2,b}

Sr. Editor. Una de las enfermedades zoonóticas con alta prevalencia en zonas tropicales en vías de desarrollo, sobre todo después de abundantes precipitaciones es la leptospirosis. La Leptospira se concentra principalmente en la orina, utilizando como reservorio principalmente a ratas, ganado y perros; parte del ciclo vital de este organismo está en el recurso hídrico, y es una patología que es «soportada por el agua», la incidencia es progresiva en zonas pobres

\footnotetext{
1 Universidad de San Martín de Porres. Filial Norte. Chiclayo, Perú.

2 Hospital Almanzor Aguinaga Asenjo - EsSalud. Chiclayo, Perú.

a Estudiante de medicina; ${ }^{b}$ Médico epidemiólogo

Recibido: 19/11/2018 Aprobado: 27/02/2019 En línea: 20/03/2018
}

Citar como: Herrera-Perez D, Saavedra-Barón A, Fernandez-Mogollon J. Leptospirosis y coinfecciones durante el niño costero en un hospital del norte peruano. Rev Peru Med Exp Salud Publica. 2019;36(1):148-50. doi:10.17843/ rpmesp.2019.361.4068 